\title{
Improved algorithm to reconstruct the thermal conductivity depth profile in hardened steels
}

\author{
by R. Celorrio*, E. Apiñaniz** A. Mendioroz ${ }^{* *}$, A. Salazar* and A. Mandelis*** \\ *Departamento de Matemática Aplicada, EUITIZ, Universidad de Zaragoza, Campus Río Ebro, Edificio Torres \\ Quevedo, 50018 Zaragoza, Spain, celorrio@unizar.es \\ **Departamento de Física Aplicada I, Escuela Técnica Superior de Ingeniería, Universidad del País Vasco, \\ Alameda Urquijo s/n, 48013 Bilbao, Spain, agustin.salazar@ehu.es \\ ${ }^{* * *}$ Center for Advanced Diffusion-Wave Technologies, Department of Mechanical and Industrial Engineering, \\ University of Toronto, Toronto, Ontario, M5S 3G8, Canada, mandelis@mie.utoronto.ca
}

\begin{abstract}
We have developed an algorithm for the accurate reconstruction of thermal conductivity depth profile of hardened steels by adding some improvements to the least square fitting, which we have used as a basis of the inversion method: (a) a renormalization of the experimental data, (b) a frequency weighting factor, (c) simultaneous fitting of amplitude and phase data, (d) a modified Tikhonov regularization procedure and (e) the Morozov discrepancy principle to stop the iterative process. The validity of the method has been tested by reconstructing theoretical data. Finally, we have retrieved the thermal conductivity depth-profile from real data.
\end{abstract}

\section{Introduction}

Depth profilometry is a very important thermal wave inverse problem where the thermal diffusivity profile is reconstructed from the measurement of the surface temperature. Thermal diffusivity is a transport property which depends on the microstructure of the material, therefore it can be used to identify changes that take place as a result of bulk modification processes such as case hardening.

In carburized and hardened steels microhardness testing is the most common technique to analyze the case depth. However, it is a destructive, time-consuming and expensive process. Since an anticorrelation between thermal diffusivity and hardness has been established, photothermal techniques provide a non-destructive method for monitoring hardness indirectly [1].

Modulated photothermal radiometry is a well established tool for the thermophysical characterization and the nondestructive evaluation of a wide variety of materials and it has been applied to reconstruct the thermal conductivity depth profile of case hardened steel pieces from surface temperature data [2,3]. This inverse problem is ill-posed in Hadamard sense, i.e. there is no continuous relationship between errors in the data and in the thermal conductivity. This means that the same surface temperature is obtained from quite different thermal conductivity profiles (within experimental uncertainty). That is the reason why the reconstructed conductivity is extremely sensitive to noise. The ill-posedness of the problem can be attenuated by reducing the number of unknowns. Under these conditions a regular least-square fitting procedure can be used successfully to retrieve the few unknowns. However, as the number of unknowns in the model is increased (in order to improve the spatial resolution of the conductivity depth profile), the problem becomes severely ill-posed and more elaborated inversion procedures need to be used such as stabilized least-square fitting, neural networks and genetic algorithms [4].

The aim of this work is to improve previous inverse methods to obtain a thermal conductivity depth profile in case hardened steel plates combining high in-depth spatial resolution and high accuracy. To do this we have developed an inverse algorithm based on an advanced least-square fitting of the surface temperature including several improvements that we will shortly describe in the next section.

\section{Results and conclusion}

By fitting surface thermal-wave data (phase and amplitude) we have successfully solved the problem of retrieving the in-depth thermal conductivity profile of heterogeneous steels. We have used the quadrupole method [5] and applied an inversion procedure based on a stabilized least-squares fitting method, to which we have applied several improvements. First, we have proceeded to perform a renormalization of the experimental data in order to eliminate experimental constants affecting both the amplitude and the phase. Second, we have combined the amplitude and phase data for the minimization and have balanced both according to their respective noise levels. This allows to penalize the magnitude with the larger noise, i.e., we increase the significance of the magnitude with less uncertainty in the inversion. Third, the introduction of a 
frequency weighting factor penalizing the influence of the high frequencies and increasing that of the low frequencies allows retrieving a realistic conductivity profile that was impossible to obtain without using the frequency weighting factor. Fourth, the ill-posed character of the inverse problem requires the use of a regularization term in the minimization of the residual. We have chosen a modification of the Tikhonov penalty term in order to stabilize the inversion. Finally, we have used the Morozov discrepancy principle to stop the iterations automatically i.e. the iterative process is stopped when the residual reaches the noise level, avoiding the "overfitting" of the data.

The validity of the method we have developed has been analyzed by retrieving conductivity profiles from noisy synthetic data generated from an exponentially decreasing conductivity profile for which analytical solution exists [6]. Finally, we have used photothermal radiometric measurements performed on several hardened AISI-1018 steel slabs to reconstruct their thermal conductivity depth profile. In figure 1 we show by symbols the normalized amplitude and phase of the surface temperature as measured by a photothermal radiometric setup. The continuous lines correspond to the fitted data using our improved inversion algorithm. The reconstructed conductivity profile (see figure 2) is in good qualitative anticorrelation with the hardness depth profile (see figure 9 in [7]), which has been obtained from mechanical indentation tests, confirming the validity of the proposed inversion procedure.

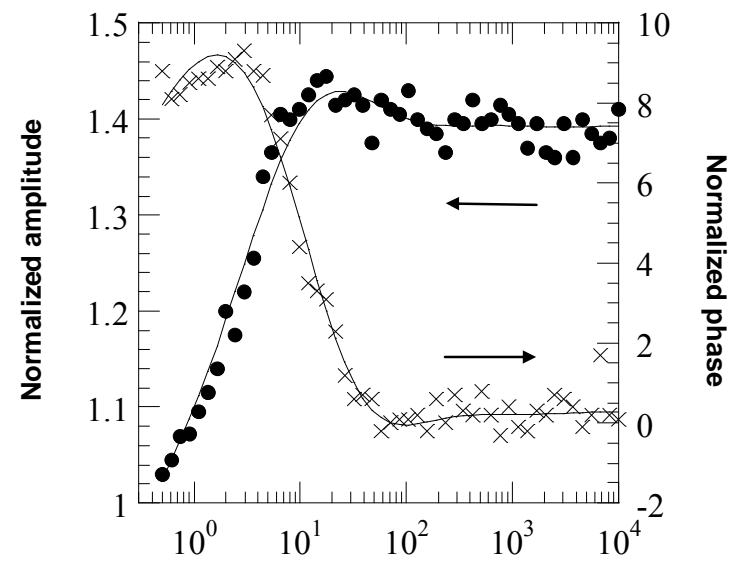

$f(\mathrm{~Hz})$

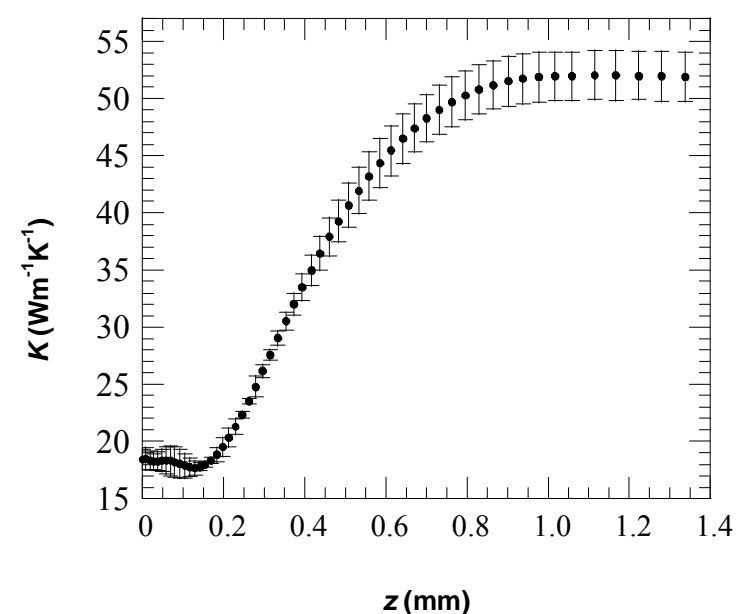

Fig. 2. Thermal conductivity reconstruction from the data depicted in figure 1.
Fig. 1. Fitting (solids lines) of the experimental amplitude (dots) and phase (crosses) data corresponding to a hardened AISI 1018 steel flat semi-infinite sample.
This work has been supported by the Ministerio de Educación y Ciencia (MAT2008-01454).

\section{REFERENCES}

[1] Almond D.P. and Patel P.M., "Photothermal Science and Techniques", Chapman and Hall,London, 1996.

[2] Munidasa M., Tian-Chi M., Mandelis A., Brown S.K. and Mannik L., Mater. Sci. Eng. A 159, 111 (1992).

[3] Mandelis A., Funak F. and Munidasa M., J. Appl. Phys. 80, 5570 (1996).

[4] Özisik M.N. and Orlande H.R.B., "Inverse Heat Transfer: Fundamentals and Applications", Taylor \& Francis, New York, 2000.

[5] Maillet D., André S.,. Batsale J.C., Degiovanni A. and Moyne C., "Thermal Quadrupoles”, Wiley, New York, 2000

[6] Fivez J. and Thoen J., J. App. Phys. 78, 2225 (1996).

[7] Wang C., Mandelis A., Qu H. and Chen Z., J. Appl. Phys. 103, 043510 (2008). 